\title{
TEORÍAS IMPLÍCITAS: SU NATURALEZA CONTEXTUAL Y PRÁCTICA COMO SABER DOCENTE PROFESIONAL
}

\author{
Denisse Esteli Núñez Ayala* \\ Universidad Autónoma de Sinaloa
}

\section{RESUMEN}

El presente artículo expone la revisión teórica sobre algunos planteamientos centrados en el ámbito del pensamiento profesional de los profesores; específicamente, de aquéllos que invitan al análisis y reflexión de sus teorías en uso, denominadas así por Argyris (como se citó en Pérez-Gómez, Soto y Serván, 2015), de naturaleza implícita (Marrero, 1988), como saberes construidos en contextos de práctica docente. En este énfasis orientado a la exploración de las teorías - como conocimiento profesional práctico-, el análisis de los procesos reguladores de la acción provocaría nuevos modos de pensar y hacer la intervención educativa en el aula tomando en cuenta, y pensando, siempre, en los alumnos y alumnas que deseamos educar y formar.

PALABRAS ClaVE: teorías implícitas, conocimiento práctico docente, pensamiento profesional.

IMPLICIT THEORIES: ITS CONTEXTUAL NATURE AND

PRACTICE AS PROFESSIONAL TEACHING KNOWLEDGE

\section{Abstract}

The present article exposes the theoretical revision on some expositions centered in the scope of the professional thought of the professors; specifically, those that invite the analysis and reflection of their theories in use, named for Argyris, (as cited in Pérez-Gómez, Soto and Serván, 2015) of implicit nature (Marrero,1988) as knowledge constructed in contexts of teaching practice. In this emphasis oriented to the exploration of the theories -the analysis of their action- regulating processes, would provoke new ways of thinking and making the educational intervention in the classroom taking into account, and always thinking about, the students that we wish to educate and train.

KEYwORDs: implicit theories, teaching practical knowledge, professional thinking. 


\section{INTRODUCCIÓN}

La construcción de teorías implícitas es una función invariante de los sujetos. Inscritos en el paradigma de la psicología cognitiva, se dice que, sin distinción alguna, todos generamos un modelo de análisis dinámico que nos permite interpretar sucesos, predecir comportamientos y tomar la decisión de actuar de una manera y no de otra; esto es, porque nos guiamos por una teoría implícita que marca las pautas de nuestra conducta (Marrero, 1988; Marrero, 2009; Rodrigo, 1985; Rodrigo, Rodríguez y Marrero, 1993).

En el caso del profesorado, éstas desempeñan un papel importante como orientadoras de la práctica y saber docente profesional. Están presentes en el diseño de la enseñanza, en las tareas académicas, en los procesos de evaluación y en la toma de decisiones que regulan los procesos educativos (Marrero, 2009).

Sin embargo, el carácter implícito y no accesible a la conciencia hace que las teorías sean resistentes al cambio y que estén fuertemente arraigadas en el sistema de creencias de los sujetos (Pajares, 1992), evitando con ello el análisis de la práctica y la reflexión sobre la acción que postula Schön (1998).

La reflexión sobre la práctica representa la oportunidad que tiene el profesional de la enseñanza para hacer explícitas sus teorías en uso (Pérez-Gómez, Soto Gómez y Serván, 2015) imprimiéndoles análisis técnico y social a los procesos educativos y, a su vez, pensando en la formación y desarrollo del alumnado que quiere educar. Hacer esto exige que el profesorado piense en la educación como «un acto de intervención en el mundo que procura cambios radicales en la sociedad» (Freire, 1997, p. 103).

Los estudios que han abordado el funcionamiento de los conocimientos cotidianos o de sentido común, en tanto elaboraciones individuales, han utilizado la categoría de teoría implícita para su análisis (Castorina, Barreiro y Toscano, 2005). Estos tienen su antecedente en la psicología popular (Marrero, 1988; Rodrigo, 1985; Rodrigo, Rodríguez y Marrero, 1993; Rodríguez y González, 1995), en los aportes de López (1989) en el caso contra la psicología popular, y de Rivière (1989) en su debate a favor de la misma. Particularmente, este último autor enfatiza en la psicología popular resaltando dos ámbitos de la misma: 1) el que denomina cinturón externo de nuestras creencias psicológicas cotidianas y es constituido por las opiniones, juicios y valoraciones que dependen, en gran medida, de las condiciones socioculturales y 2) el núcleo duro de la psicología popular, comprendido por un conjunto de principios de funcionamiento psicológico que están implícitos en nuestros usos lingüísticos habituales y que dirigen nuestras interacciones con los

*E-mail: denisssenunez@uas.edu.mx. Es licenciada en Educación por la Universidad Pedagógica del Estado de Sinaloa, maestra en Educación y doctora en Educación por la Universidad Autónoma de Sinaloa. Tiene como línea de investigación la formación inicial y el desarrollo profesional docente con interés particular en la educación inclusiva y el estudio de las teorías implícitas sobre diversidad en profesores de educación básica. 
(Rivière, 1989). Dentro de estas temáticas, la revisión de la literatura especializada advierte la presencia de dos líneas de investigación que brindan elementos teóricos y empíricos para estudiar cómo los sujetos interpretan y organizan su realidad del mundo. La primera enfatiza en el modelo socioconstructivista para la construcción del conocimiento individual (Marrero, 1988; Rodrigo, Rodríguez y Marrero, 1993); y la segunda, en las representaciones para el análisis de las teorías implícitas (Pozo, 2006; Pozo, Scheuer, Pérez y Mateos, 2006). Ambas, desde la psicología cognitiva, sin embargo, metodológicamente diferentes para abordar al objeto de estudio en su complejidad.

Haciendo énfasis en la primera línea de investigación, es posible identificar la existencia de posiciones teóricas distintas. Por una parte, la individual considera que el conocimiento es producto del pensamiento eminentemente psicológico, derivado del funcionamiento del sistema cognitivo, y, por la otra, la cultural afirma que es la intervención del sujeto dentro del sistema y los grupos sociales lo que permite la emergencia del conocimiento como producto eminentemente social (Marrero, 1988; Rodrigo, 1985; Rodrigo, Rodríguez y Marrero, 1993).

Ubicados en la postura que recupera el carácter sociocultural, es válido señalar que, sin importar la cultura, todos los sujetos como agentes activos generamos teorías implícitas. Es una función propia de la especie humana y, además, viene garantizada por medio de tres tipos de anclajes básicos: biológico, social y representacional; definidos éstos por Rodrigo, Rodríguez y Marrero (1993) en su obra clásica Las teorías implícitas. Una aproximación al conocimiento cotidiano.

Esta posición -la cultural-interpreta a las teorías como unidades explicativas de conocimiento social, que se abstraen como resultado de las experiencias episódicas del sujeto referentes a un dominio específico (Marrero, 1988). Entendiéndolo así, son experiencias almacenadas en categorías de información que dan cuenta de la misma como respuesta al contexto.

Son teorías porque han sido elaboradas individualmente por el sujeto como modelo interpretativo de su mundo. Además, porque reúnen un conjunto de contenidos culturales; organizados sistémicamente y con cierta recurrencia, en ideas típicas y representativas, sobre determinado ámbito de la realidad. Aunado a esta idea, y bajo el planteamiento de Rivière (1987), también se dice que «tienen limites difusos y sus elementos no son equivalentes entre sí, sino que definen un continuo de tipicidad o representatividad, de forma que determinados ejemplares son más prototípicos del concepto definido" (p. 14). Con ideas similares, Marrero (1988) advierte que sus limites son borrosos y algunas experiencias que sirven de base para la extracción de una teoría pueden constituir también elementos indispensables en otra. Así, debido al contenido cultural la construcción de teorías no es totalmente pura, ni tampoco aparece de forma súbita; por el contrario, es resultado de la participación por diversos ámbitos de experiencias.

Se les denomina implícitas porque no son accesibles a la conciencia, el sujeto portador de teorías no sabe cómo llegó a ellas y no dispone de una formulación verbal para explicitarlas; no obstante, una vía para su explicitación podría ser a través de procesos de análisis consciente, recuperando el curso vivido del sujeto; también puede ser inferida por el observador o investigador (Marrero, 1988; Rodrigo, Rodrí- 
guez y Marrero, 1993). De esta manera, un rasgo característico de dichas teorías es el carácter implícito que las hace resistentes al cambio y, debido a ello, permanecen incrustadas en el sistema de creencias del sujeto, característica por lo cual Marrero (2009) las sitúa en el «territorio cognitivo pantanoso, ambiguo, móvil y líquido que está en constante reestructuración -adaptación cognitiva-» (p. 250).

En suma, la entidad representacional de las teorías implícitas o también denominadas teorías en uso por Argyris (como se citó en Pérez-Gómez, Soto y Serván, 2015) es la apropiación que el sujeto hace de la cultura. Ésta última proporciona los códigos culturales que son expuestos al mundo de forma inconsciente, a través de las acciones y emociones que regulan la conducta y el comportamiento individual. Por tanto, las teorías implícitas son el vínculo más fiel entre la cultura y el pensamiento humano (Marrero, 1988).

\section{LA NATURALEZA CONTEXTUAL DE LAS TEORÍAS IMPLÍCITAS}

Sin olvidar al sujeto de la psicología cognitiva portador de teorías (Rivière, 1987), la dimensión cultural es un componente imprescindible para el diseño de las mismas (Marrero, 1988; Rodrigo, Rodríguez y Marrero, 1993). Es así porque son «el resultado de una delicada interacción entre las estrategias individuales de procesamiento de información y los procesos socioculturales a gran escala» (Rodrigo, 1985, p. 146). Además, como mencionan Pozo, Scheuer, Mateos y Pérez (2006), «las heredamos sin testamento, sin que seamos conscientes con frecuencia de lo que estamos heredando y, por tanto, sin que podamos resistirnos a esa herencia o cambiarla» (p. 101). De esta manera, el sujeto desconoce el origen contextual de sus teorías; por ello, «las personas que hacen un uso pragmático de ellas no pueden, sin embargo, hacer un uso epistémico pleno de las mismas, es decir, no tienen conocimiento, en parte o en todo, de esas teorías para representarse el mundo» (Pozo, 2006, p. 113).

Por otra parte, para entender la naturaleza representacional del sujeto, De Vega (1985) cuestiona ¿cómo sería el diseño cognitivo de una especie cultural? En su respuesta, afirma que se esperaría un conjunto de rasgos bastante heterogéneos, porque una especie que genera y transmite cultura debe poseer representaciones funcionales que permitan «simular» estados posibles del mundo. Siguiendo la idea del autor, es preciso señalar que la cultura produce enriquecimiento de las funciones mentales de la especie. En consecuencia, «las teorías se construyen a partir de experiencias directas en contextos culturales, compartidas o no con los otros, o bien, a partir de las experiencias obtenidas durante la observación del comportamiento ajeno (Rodrigo, Rodríguez y Marrero, 1993, p. 59).

Desde los planteamientos antes mencionados, el marco de acción de las teorías implícitas es el modelo representacional de los sujetos; es éste el que gobierna la acción, permitiendo que determinados contenidos sociales se inserten en la mente de las personas como prototipos culturales. Dicho así, lo cierto es que no existen las teorías implícitas por sí mismas. Lo que prevalece son las experiencias que los sujetos recuperan en su trayecto e intervención directa por los contextos (Marrero, 1988). 
De ahí que éstas se organizan y funcionan como síntesis de ideas ordenadas, en función del principio de tipicidad, polaridad o grado de semejanza con otras teorías que mejor responden a la tarea o actividad (Marrero, 1988; Rodríguez y González, 1995).

Es válido resaltar que la naturaleza de las teorías implícitas es plural, heterogénea y compleja. Recupera tanto la dimensión cultural como la psicológica para generar un concierto dinámico de ideas inscritas en el sistema de creencias de las personas. Centrados en esta postura, Rodrigo (1985) ha dicho que se requieren ambas dimensiones para poder dar una imagen más completa de lo que son las teorías implícitas. Por esta razón, es bueno recordar que son contenidos sociales, que se oponen al reduccionismo y simplicidad que separa y aísla posiciones teóricas para el estudio del pensamiento humano (Morin, 2002); tal cual se demuestra en la postura individual de Piaget y la social de Moscovici, para comprender el proceso de construcción sociocultural de las teorías implícitas.

Por tanto, el sujeto de la psicología cognitiva (Rivière, 1987) generador de teorías hace uso de todos aquellos productos culturales que son resultado del proceso de socialización (Berger y Luckman, 1986) tales como emociones, lenguaje, expresiones y todo cuanto está dispuesto y expuesto en la cultura. Ello indica que mientras más ricos sean los contextos en los que se interviene, mayor es el potencial de significaciones para representarse la realidad social.

\section{LAS TEORÍAS IMPLÍ́CITAS Y EL SABER DOCENTE PROFESIONAL}

Los estudios inscritos en la línea del pensamiento del profesorado centran su atención en describir la vida mental del mismo para comprender el cómo y el porqué de sus actividades profesionales (Contreras, 1985). En ese sentido, "son un ejemplo de cómo el estudio de la psique individual, de sus mecanismos y peculiaridades puede ayudar y ser un elemento esencial para la comprensión de lo educativo» (Marrero (2009, p. 23). Ubicados en esta línea, Pérez-Gómez (1983), por su parte, sitúa el análisis de la investigación sobre el pensamiento del profesorado en un modelo ecológico del aula que explica tanto las redes de intercambio como los significados producidos en el contexto de las exigencias institucionales y que, además, median el modo de orientar los procesos educativos. Con planteamientos similares y cuestionando el carácter meramente cognitivo de las teorías implícitas, Marrero (2009) advierte que prevalece un vínculo importante entre la cultura y la acción educativa; o bien, que el "pensamiento docente es una síntesis entre los aspectos individuales y culturales de la educación» (Marrero, 1988, pp. 70-71). Por esta razón, este último autor, situado en el modelo socioconstructivista, se planteó abrir las fronteras mentalistas entre lo psicologista y sociologista en la concepción, interpretación y análisis del pensamiento del profesor. Por consiguiente, destacando la síntesis entre lo social y cultural, puntualiza definiendo a las teorías implícitas docentes como

síntesis de experiencias prácticas pedagógicas que se construyen en el escenario de la cultura escolar y engloban diversos tipos de "conocimiento" -sobre la materia, sobre la gestión del aula, valorativo, sobre el alumnado-, «conocimiento personal 
práctico»-reglas, principios prácticos, imágenes, dilemas, metáforas, ritmos...- y «creencias»-decisiones, acciones, rutinas, hábitos...- que se traducen en «esquemas de actuación y comprensión» que guían la «acción práctica» (Marrero, 2009, p. 37).

Lo antes expuesto invita a enfatizar en las teorías implícitas del profesorado como el saber profesional que se produce en contextos de enseńanza; que son resultado de las experiencias directas que se abstraen en relación con la intervención pedagógica y, además, que orientan el marco interpretativo para la acción; son un tipo de lenguaje común, implícito, que está latente y caracteriza las prácticas educativas, y, por último, también son responsables de la automatización de las acciones recursivas que se muestran resistentes al replanteamiento de las actividades de enseñanza (Marrero, 1988; Rodrigo, 1985; Rodrigo, Rodríguez y Marrero, 1993).

Recuperando la dimensión cultural, es posible señalar que la teoría implícita se va nutriendo durante los años de escolarización que se pasan como estudiante, o bien en la etapa preformativa y en la formación inicial como profesionales de la educación (Jacobo, 1997, 2009; Marcelo, 1995; Pajares, 1992). Desde ese momento, el sujeto va generando un saber ser y saber hacer en relación con el ejercicio de la profesión y que, indudablemente, se activará ante ciertas demandas socioculturales de la acción profesional.

Por otra parte, para Porlán, Rivero y Martín (1997) el pensamiento del profesorado resulta de un nuevo conocimiento profesional; práctico, pero no académico, aunque toma en consideración los aportes de diversas disciplinas. Tampoco es empírico, aunque se basa en la experiencia para intervenir en ella. Es un conocimiento integrador y profesionalizado que se organiza en torno a los problemas de la práctica; se construye en relación con las actividades de formación en las que participa y, además, «es contextualizado, social y distribuido» de acuerdo con Kincheloe, Steinberg y Villaverde (como se citó en Marrero, 2009, p. 33).

Sin duda, el conocimiento práctico profesional docente es experiencial y se construye de manera situada en los contextos. Es precisamente en esos lugares de intervención pedagógica donde el profesorado genera su saber, gracias tanto al compromiso emocional que tiene con su profesión como a toda la actividad mental implicada en el procesamiento de la información, de su entorno escolar y comunitario. Es bueno decir que ahí los profesores aprenden, a la vez que enseñan (Marcelo, 1988; 1991; Feiman-Nemser, Schwille y Carver, 1999). Por ello, como dice Marrero (2009), «el conocimiento en general y el pedagógico en particular no puede comprenderse al margen del contexto en el que surge y al que se aplica» (p. 34). Sin embargo, los planteamientos de Vaillant y Marcelo (2015) se inclinan por declarar que «las relaciones entre los Centros Educativos y las Instituciones de Formación -en la formación inicial- han estado caracterizadas por el desencuentro, un cuasi divorcio o, simplemente, por mutua ignorancia» (p. 64).

Dentro de este ámbito de estudio, para Pérez-Gómez, Soto y Serván (2015) el conocimiento práctico docente engloba connotaciones emotivas, es de carácter holístico, emergente, funcional, inconsciente e intuitivo. Además, lo caracterizan como de tipo experiencial, situacional y contextualizado porque se produce en escenarios de práctica pedagógica. Asociado a esta idea, los autores arriba citados también 
señalan que los docentes no son conscientes de la naturaleza de su conocimiento; no obstante, continuamente están haciendo uso del mismo y lo activan en cada situación concreta durante la enseñanza.

Con posiciones similares que recuperan las connotaciones emotivas del conocimiento práctico docente, Clarà y Mauri (2010) y Pérez-Gómez (2010) señalan que éste activa un saber ser, sentir y hacer que representa a la dimensión personal del profesor. Estos planteamientos se ubican en la línea de estudio de Elbaz (2006) y Clandinin y Connelly (1987) destacando que cuando los profesores actúan como profesionales también son personas; que hacen uso de sus emociones, afectos, creencias, valores y actitudes como orientadoras de su comportamiento. Por ello la condición encarnada de las teorías (Pozo, 2006) resistentes al cambio; porque derivan de un tipo de conocimiento que se produce en la acción y operan de manera automática, sin necesidad de la conciencia.

Asociado a la idea anterior, otros autores han dicho que si no se modifican las teorías arraigadas y expresadas en los comportamientos individuales, no será posible la transformación real de los profesionales innovadores (Marrero, 2009; Pérez-Gómez, 2013). Quizá porque, como mencionan Marcelo y Vaillant (2009), el cambio de creencias es un proceso lento y, en relación con Guskey y Sparks, «las creencias cambian no como consecuencia de participar en actividades de desarrollo profesional, sino a partir del contraste con las nuevas prácticas que se proponen desarrollar» (como se citó en Marcelo y Vaillant, 2009, p. 69).

Por ello, para romper con la causalidad lineal y reproducción de teorías (Morin, 2002) es necesario que el profesorado recupere sus experiencias pedagógicas de manera reflexiva; que revise y sustente sus acciones desde la racionalidad técnica que plantea Schön (1998) y desde las dimensiones de la reflexión práctica, técnica y social para el ejercicio de la profesión que sugiere Jacobo (2009). En esta misma línea, Korthagen (2010) coincide con la idea de que los profesores reflexionen constantemente, sobre una clase, un alumno específico, su comportamiento educativo o sus competencias.

Asimismo, es importante decir también que para Dewey (2007) la reflexión trae consigo, siempre, una consecuencia u ordenación consecuente de ideas. Es, entonces, la que les da sentido a las decisiones docentes y, por ello, representa una actividad mental importante para todo aquel profesional interesado en la revisión de sus procesos de enseñanza; contrastando visiones, maneras de ser y hacer el ejercicio docente, para su reconstrucción. De igual forma, Freire (1997) le adjudica valor a la reflexión, nombrándola como uno de los «saberes necesarios para la práctica educativa»; esta idea la puntualiza el autor diciendo que «es pensando críticamente la práctica de hoy o la de ayer como se puede mejorar la próxima» (p. 40). Aunado a estas ideas, desde los planteamientos teóricos de Pérez-Gómez, Soto y Serván (2015), es a partir de la reflexión como el conocimiento práctico se transforma en pensamiento práctico, que resulta de la teorización. Además, siguiendo con los autores, provocaría que «se identifiquen, analicen y reformulen no sólo las teorías proclamadas que adornan el discurso, sino también las teorías en uso que gobiernan la práctica» (Pérez-Gómez, Soto y Serván, 2015, p. 85). 
Asimismo, para Pozo, Martín y Pérez (2010) la reflexión es insuficiente para reconstruir la práctica docente. En cambio, advierten que «no basta con hacer explícito lo implícito, necesitamos también hacer implícito lo explícito, porque sólo cuando ese conocimiento teórico se formatee como acciones, adopte el formato de la acción (situado, encarnado, implícito) podrá ser realmente eficaz» (p. 183). Esto refiere a que el cambio de teorías habrá de conseguirse modificando profundamente la base psicológica que guía la acción y representación de las mismas.

Por otra parte, no olvidemos que las teorías implícitas del profesorado son el resultado de muchas síntesis posibles: experiencias y acciones acumuladas derivadas de los espacios socioculturales en los que intervienen (Marrero, 2009). Entendido esto, y como resultado de la dimensión cultural, el saber del profesorado es policéntrico porque está organizado en torno a epistemologías diversas (Hernández, 2009).

Por tanto, conocer la naturaleza y los contenidos de las teorías implícitas del profesorado y, sobre todo, cómo éstos orientan su intervención pedagógica tendría implicaciones importantes en la formación y desarrollo profesional docente. En nuevos modos de pensar la profesión. Ello requiere recuperar los saberes profesionales que tienen origen en los contextos escolares para establecer vínculos significativos entre la teoría y la práctica. O bien, una estrecha y continua relación. En esta idea, menciona Darling-Hammond (2012):

Para que los futuros docentes puedan tener éxito en su labor, los centros de formación deben diseñar programas que cambien los parámetros con los cuales los novatos aprenden a enseñar y luego se vuelven profesores. Esto significa que la formación docente debe aventurarse cada vez más lejos de la universidad y acercarse a las escuelas en una agenda de transformación mutua con todo el esfuerzo y la complejidad que ello implica (p. 18).

Es necesario repensar los espacios donde se aprende a enseñar. Con ideas similares a la autora, Jacobo y Pintos (2003) han dicho que la formación profesional no debería realizarse más en las Instituciones Formadoras y Actualizadoras de Docentes (IFAD) con independencia técnica de las escuelas de educación básica y de la cultura de las comunidades en las que éstas se ubican. Se requiere innovar, afirman, para ir en búsqueda de nuevos escenarios para la formación de los educadores mexicanos. Siguiendo el planteamiento de Jacobo y Pintos (2003), «se necesitaría recrear el sistema educativo nacional para que quienes reciben la formación inicial como docentes y los que se encuentran en servicio, desaprendan todas aquellas creencias y estilos cognitivos identificados con la educación en la edad de las máquinas -industrial-y para que reaprendan, en cambio, las que demanda la educación en la edad de los sistemas, la era de la información y del conocimiento» (p. 29). Se requiere que los profesionales de la educación comprendan la enseñanza y el aprendizaje para un mundo cambiante (Darling-Hammond, 2012). No olvidando, además, que, como lo plantea Freire, «mi práctica profesional, que es la práctica docente, al no ser superior ni inferior a ninguna otra, exige de mí un alto nivel de responsabilidad ética de la cual forma parte de mi propia capacitación científica» (p. 134). 
Finalmente, debemos decir que las teorías implícitas del profesorado, por tener un carácter contextual y a la vez heterogéneo, recuperan los significados y demandas educativas inscritas en los espacios escolares. En por ello por lo que invitan a pensar nuevos modos de propiciar la formación profesional docente, para que los centros escolares puedan responder a la demanda y expectativas de las comunidades en las que se insertan. En esta idea, no olvidemos que la formación profesional, en el caso de los profesores, está desbordándose, sucede cada vez menos en las escuelas normales como espacios tradicionales (Jacobo, 2009).

Con la presente revisión teórica se propuso resaltar algunos de los planteamientos que recuperan al profesorado como sujeto de saber; con capacidad para el análisis de los contenidos que gobiernan su pensamiento característico de su profesión. Indagar en estos temas, es decir, saber qué piensan los profesores, cómo actúan y por qué lo hacen de una manera y no de otra; o bien, hacer explícito cómo resuelven los problemas de sus prácticas, "permitiría observar cómo surge toda una serie de representaciones sobre las políticas para reformar, reestructurar y reconceptualizar la educación» (Marrero, 2009, p. 37). 


\section{REFERENCIAS BIBLIOGRÁFICAS}

Berger, P. y Luckmann, T. (1968). La construcción social de la Realidad. Argentina: Morata.

Castorina, J., Barreiro, A. y Toscano, A. (2007). «Dos versiones del sentido común: las teorías implícitas y las representaciones sociales», cap. VIII, en CASTORInA, J. Construcción conceptual y representaciones sociales. El conocimiento de la sociedad. Argentina: Miño y Dávila.

Contreras, D. (1985) «¿El pensamiento o el conocimiento del profesor? Una critica a los postulados de las investigaciones sobre el pensamiento del profesor y sus implicaciones para la formación del profesorado". Revista de educación, 277, 19, recuperado de https://dialnet.unirioja.es/ servlet/autor?codigo $=18229$.

Darling-Hammond, L. (2012). Educar con calidad y equidad. Los dilemas del siglo XXI. Santiago de Chile: Centro de Innovación en Educación de Fundación Chile.

Dewey, J. (2007). Cómo pensamos. La relación entre pensamiento reflexivo y proceso educativo. España: Paidós.

Feiman, N.S., Schwille, S., Carver, C. y Yusko, B. (1999). «A conceptual Review of literature on new teacher induction». National Partnership for Excellence and Accountability in Teaching, Washington, DC, recuperado de https://eric.ed.gov/?id=ED449147.

Freire, P. (1997). Pedagogía de la autonomía. Saberes necesarios para la práctica educativa. México: Siglo XXI.

Hernández, A. (2009). «Mutaciones en el mundo, visiones del profesor: itinerario conceptual para repensar el saber docente», en Marrero, J. (2009). El pensamiento reencontrado. España: Octaedro.

JАСово, Н.M (1997). La Exploración del pensamiento pedagógico de los profesores de educación básica: una contrastación con los pedagogos clásicos del primer tercio del presente siglo (XX). (Tesis doctoral), Universidad Autónoma de Madrid, Madrid, Espańa.

Jасово, H.M у Pintos, J.L. (2003). Nuevos escenarios en la formación de los educadores mexiacanos. Una visión sistémica. México: SEP/ CONALITEG.

Јасово, Н. М (2009). El profesionalismo integrado. Un nuevo modo de ser educador. México: Plaza y Valdés.

Korthagen, F. (2010). «La práctica, la teoría y la persona en la formación del profesorado». Revista Interuniversitaria de Formación del Profesorado, 24, (2), 83-101, recuperado de http://www. redalyc.org/articulo.oa?id=27419198005.

López, C. (1989). «El caso contra la psicología popular». Cognitiva, 2(3), 227-242.

Marcelo, C. y Vaillant, D. (2009). Desarrollo profesional docente ¿Cómo se aprende a enseñar? España: Narcea.

Marrero, J. (1988). Teorias implícitas y planificación del profesor. (Tesis doctoral). Universidad de La Laguna. Santa Cruz de Tenerife, España.

Marrero, J. (2009). El pensamiento reencontrado. Espańa: Octaedro.

Morin, E. (ed.) (2002). La mente bien ordenada. España: Seix Barral.

Pajares, M.F. (1992). «Teachers' Beliefs and Educational Research: Cleaning Up a Messy Construct». Review of Educational Research, 62(3), 307-332, recuperado de https://doi. org/10.3102/00346543062003307. 
Pérez-Gómez, Á. (2010). "The nature of the practical knowledge and its implications in teacher training». Infancia y Aprendizaje, 33(2), 171-177, recuperado de https://doi. org/10.1174/021037010791114652.

Pérez-Gómez, Á., Soto-Gómez, E., Servan-NúÑez, M. (2015). «Lesson Studies: re-pensar y re-crear el conocimiento práctico en cooperación». Revista Interuniversitaria de Formación del Profesorado, 29(3) 81-101, recuperado de http://www.redalyc.org/articulo.oa?id=27443871006.

Porlán, A.R., Rivero, G.A. y Martín, R. (1997). «Conocimiento profesional y epistemológico de los profesores I: Teoría, métodos e instrumentos». Enseñanza de las ciencias, 15 (2), 156.

Pozo, J.I. (2006). Adquisición del conocimiento. España: Morata.

Pozo, I., Martin, E., Pérez-Echeverría, M.P., Scheuer, N., Mateos, M. y de la Cruz, M.. (2010). «Ni contigo, ni sin ti. Las relaciones entre cognición y acción en la práctica educativa». Infancia y Aprendizaje, 33, 179-184, recuperado de https://doi.org/10.1174/021037010791114580.

Rivière, A. (1987). El sujeto de la Psicología Cognitiva. Madrid: Alianza.

Rivière, A. (1989). «Más a favor de la psicología popular». Cognitiva, 2(3), 261-265.

Rodrigo, M.J. (1985). Las teorías implícitas en el conocimiento social. Infancia y aprendizaje, 8(3132), 145-156.

Rodrigo, M.J., Rodríguez, A. y Marrero, J. (1993). Las teorias implícitas: una aproximación al conocimiento cotidiano. Madrid: Visor.

SснӧN, D. (1998). El profesional reflexivo. Cómo piensan los profesionales cuando actúan. España: Paidós.

TARDif, M. (2004). Los saberes del docente y su desarrollo profesional. España: Narcea.

Vaillant, D. y Marcelo, C. (2015) El A, B, Cy D de la formación docente. España: Narcea.

VEGA, M. de (1985). «Nuevas perspectivas del procesamiento de información». Estudios de Psicologia, 6(22), 3-17. 
